\title{
Correlation of Four Single Nucleotide Polymorphisms of the RELN Gene With Schizophrenia
}

\author{
J Ping", J Zhang*, J Wan, A Banerjee, C Huang, J Yu, T Jiang, B Du \\ * Equal contribution
}

\begin{abstract}
Objective: This study aims to determine the association between single-nucleotide polymorphisms (SNPs) of the RELN gene and schizophrenia.

Methods: 134 patients aged 16 to 58 (mean, 38.0) years who were diagnosed with acute or chronic schizophrenia at the Zhongshan Third People's Hospital between January 2018 and April 2020 were recruited, as were 64 healthy controls aged 22 to 59 (mean, 45.6) years who matched with the age and sex of the patients. MassARRAY mass spectrometry genotyping technology was used to determine the genotypes of four SNPs of RELN (rs2073559, rs2229864, rs362691, and rs736707).

Results: There were no significant between-group or between-sex differences in terms of genotype, allele frequency, or haplotype frequency of the SNPs (all p > 0.05). In the association analysis between genotypes and quantitative traits in the Positive and Negative Syndrome Scale, rs2229864 and rs736707 were associated with the scores for items P3 (hallucinatory behaviour) and G11 (attention disorder), and rs362691 was associated with G10 (disorientation). However, the associations did not remain significant after Bonferroni correction.

Conclusion: Multiple pathogenic polymorphisms of RELN might be associated with hallucinatory behaviour and attention disorder in Chinese patients with schizophrenia.
\end{abstract}

Key words: Alleles; Genotype; Hallucinations; Polymorphism, single nucleotide; Reelin protein; Schizophrenia

Junjiao Ping, MS, Joint Laboratory of Psychiatric Genetic Research and Department of Psychiatry, The Third People's Hospital of Zhongshan, Zhongshan, Guangdong, PR China

Jie Zhang, MD, PhD, Joint Laboratory of Psychiatric Genetic Research and Department of Psychiatry, The Third People's Hospital of Zhongshan, Zhongshan, Guangdong, PR China

Jing Wan, MM; Department of Early Intervention, The Third People's Hospital of Zhongshan, Zhongshan, Guangdong, PR China

Ashmita Banerjee, MD, Department of Psychiatry, University of Iowa Carver College of Medicine, Iowa City, IA, United States

Caiying Huang, MBBS, Department of Early Intervention, The Third People's Hospital of Zhongshan, Zhongshan, Guangdong, PR China

Jinming Yu, MBBS, Department of Substance Dependence, The Third People's Hospital of Zhongshan, Zhongshan, Guangdong, PR China

Tingyun Jiang, MD, Department of Psychiatry, The Third People's Hospital of Zhongshan, Zhongshan, Guangdong, PR China

Baoguo Du, MM, Department of Clinical Psychology, The Third People's

Hospital of Zhongshan, Zhongshan, Guangdong, PR China

Address for correspondence: Baoguo Du, Department of Clinical Psychology, The Third People's Hospital of Zhongshan, No. 18 of Nanqi South Road, Nanlang District, Zhongshan 528400, Guangdong, PR China.

Email:dubaoguo99@sina.com

Submitted: 24 September 2021; Accepted: 9 December 2021

\section{Introduction}

Schizophrenia is a complex genetically based psychiatric disorder characterised by auditory hallucinations, delusions, and cognitive impairment. The onset of schizophrenia usually occurs in late adolescence or early adulthood. ${ }^{1}$ Schizophrenia is one of the ten most common causes of disability in young people. Its prevalence is approximately $1 \%$. According to the 2019 mental health epidemiological survey in China, the lifetime prevalence of schizophrenia is $0.7 \% .^{2}$ Schizophrenia impacts patients' social relations and employment opportunities and is a heavy economic burden. ${ }^{3,4}$ Family, twin, and adoption studies have reported that schizophrenia has a strong genetic tendency and that its heritability can reach $80 \%$ to $85 \% .^{5}$ However, finding a susceptibility gene is difficult. At least 100 genes have been reported to be associated with schizophrenia, but only a few have consistently shown such an association in multiple regions and ethnic groups. ${ }^{6}$ Genome-wide association studies that investigate single-nucleotide polymorphisms (SNPs) and copy number variation have been used to provide genetic evidence for the aetiology of schizophrenia. Multiple functional variants of genes in neurodevelopmental pathways may be involved in the development of the illness. ${ }^{7,8}$

Brain-structure and animal experiments have revealed that aberrations in neurodevelopment may be a factor in the aetiology of schizophrenia. Moreover, molecular genetic studies have confirmed several repeatable chromosomal 
locations and susceptibility genes, suggesting that genetic variations in multiple neurodevelopmental pathways are involved in the disease progress. ${ }^{9}$

A number of susceptibility genes are implicated for schizophrenia, including several short repetitive sequences in the RELN gene, which is located on chromosome 7q22.1 and encodes the protein reelin. ${ }^{10}$ rs7341475 polymorphism increases the risk of schizophrenia by 1.4 -fold in women. ${ }^{11}$ Genetic variants in RELN are associated with working memory, situational memory, and executive function in core symptoms of patients with schizophrenia. ${ }^{10}$ SNPs in some RELNs may mediate schizophrenia pathogenesis and clinical symptom severity. ${ }^{12-14}$

Reelin is a serine protease located in the extracellular matrix. During brain development, reelin plays an important role in the migration of neurons and formation of the sebaceous layer. In mice, spontaneous deletion of RELN leads to abnormal brain function, causing uncoordinated movement. Reelin is also important for brain function after birth and throughout adulthood. Adult reelin signal interruption causes changes in synaptic signalling and plasticity, leading to neuropsychiatric diseases. ${ }^{15}$ In a Finnish population, loci in the $7 \mathrm{q} 22$ region, including $R E L N$, are associated with the endophenotype of schizophrenia. ${ }^{16}$

It remains controversial whether the genetic polymorphism of RELN is associated with psychiatric disorders. Some found evidence for the association between RELN polymorphism and schizophrenia; others reported dissimilar conclusions. In a meta-analysis of SNPs of RELN, the rs736707 locus was associated with schizophrenia in the Asian population. ${ }^{17}$ In a study of 103 schizophrenic patients and 169 healthy controls in Guangdong province, China, the A-C-T-T-A and A-C-T-T-G haplotypes in the rs362814rs39339-rs540058-rs661575-rs727708 haplotype system of $R E L N$ were associated with increased susceptibility to schizophrenia. ${ }^{18}$ In contrast, in a study of 761 schizophrenic patients and 775 healthy controls in Jilin province, China, no association was found between the RELN rs736707 locus and schizophrenia. ${ }^{19}$ The diverse findings may be due to the small sample size and/or patient heterogeneity (including sex differences or family history of illness). The present study aims to investigate associations between the rs2073559, rs2229864, rs362691, and rs736707 loci of RELN and schizophrenia in a population in Zhongshan, Guangdong, China.

\section{Methods}

This study was approved by the ethics committee of Zhongshan Third People's Hospital (reference: SSYLL20180301). Written informed consent was obtained from each participant or his guardian. 134 patients (92 males and 42 females) of Han ethnicity aged 16 to 58 (mean, 38.0) years who were diagnosed with acute or chronic schizophrenia based on the DSM-5 by two attending psychiatrists at the Zhongshan Third People's Hospital between January 2018 and April 2020 were recruited. Those with major physical diseases (eg, diabetes, hypertension, cancer), nervous system diseases, or other psychiatric diseases were excluded. In addition, 64 healthy controls (38 males and 26 females) aged 22 to 59 (mean, 45.6) years who matched with the age and sex of the patients were recruited. They were workers, nurses, volunteers, and physical examinees from the Zhongshan Third People's Hospital. Those who were adopted or from single-parent families with an unknown family history, with a history of major physical diseases (such as diabetes, hypertension, or cancer), or with a personal history or family history of mental illness were excluded.

For each participant, $5 \mathrm{~mL}$ peripheral venous blood was collected. Genomic DNA was extracted using a Tiangen Blood Genomic DNA Extraction Kit (Tiangen Biotech, Beijing, China) and stored at $-80^{\circ} \mathrm{C}$. The MassARRAY SNP genotyping platform was used for RELN genotyping. The loci rs2073559, rs2229864, rs362691, and rs736707 were input into the Agena website (http://agenacx.com/) for primer design, and the primers were ordered.

Statistical analysis was performed using SPSS (Windows version 20; IBM Corp, Armonk [NY], US). The Hardy-Weinberg equilibrium was tested using the Chi square test. The online software SHEsis was used for the pairwise SNP linkage disequilibrium, unit point, and haplotype association analyses. A p value of $<0.05$ was considered statistically significant.

Differences in the frequency distribution of the alleles, genotypes, and haplotypes of linkage disequilibrium in each SNP locus between the schizophrenia and healthy control groups were determined, as were their association with schizophrenia. The degree of linkage disequilibrium between each pair of SNPs was expressed as D' value ranging from 0 to 1 . Higher values indicate higher linkage disequilibrium between the two loci. Then, the polymorphic loci were grouped according to the $\mathrm{D}^{\prime}$ value, and the SNPs with high linkage disequilibrium were combined into a group for haplotype analysis to determine the 'pair local $\mathrm{D}^{\prime} / \mathrm{r}^{2}$ value'.

The expectation maximisation calculation method was used to estimate the haplotype frequency, and the threshold was set at 0.03 . For the haplotypes, the allele combination with the highest frequency was used as a reference and compared with other allele combinations. The significance of association between a haplotype and schizophrenia was determined by the overall $\mathrm{p}$ value.

The optical density of the genomic DNA was measured. All DNA concentrations were $>20 \mathrm{ng} / \mu \mathrm{L}$; optical density of 260/280 was between 1.6 and 2.2 and optical density of $260 / 230$ was $>0.6$. There was no absorption peak at $230 \mathrm{~nm}$. The DNA bands were intact without serious degradation.

\section{Results}

According to the Hardy-Weinberg genetic equilibrium, there was no significant difference in the gene frequency 
of the rs2073559, rs2229864, rs362691, and rs736707 loci of RELN between the schizophrenia and healthy control groups or between sexes (Tables 1 and 2). Thus, the two groups are representative of the regional population.

As the genetic distance was long between the four studied polymorphic loci of the RELN gene, the $\mathrm{R}^{2}$ value was relatively low. Therefore, the $\mathrm{D}^{\prime}$ value was used to represent the linkage disequilibrium between the loci. The $\mathrm{D}^{\prime}$ values were large between rs2073559 and rs362691 $\left(\mathrm{D}^{\prime}=0.790\right)$ and between rs2229864 and rs736707 $(\mathrm{v}=0.923)$, whereas the D' values was low between other pairs of loci $\left(\mathrm{D}^{\prime}<0.5\right)$.

Only common haplotypes with a frequency of $\geq 3 \%$ were selected to determine the association between each haplotype and schizophrenia. There were significant differences between the schizophrenia and healthy control groups in rs2073559/rs2229864 (TA*), rs2073559/ rs2229864/rs362691 (TAG*), and rs2073559/rs2229864/ rs362691/rs736707 (TAGA*), but the difference became not significant in a pairwise comparison (Table 3 ).

In the association analysis between genotypes and quantitative traits in the Positive and Negative Syndrome Scale, rs2229864 and rs736707 were associated with both P3 (hallucinatory behaviour) and G11 (attention disorder) [both $\mathrm{p}<0.05$ ], and rs362691 was associated with G10 (disorientation) $[\mathrm{p}<0.05]$. However, these associations became not significant after Bonferroni correction (Table 4).

\section{Discussion}

Reelin is a member of the glycoprotein family and is involved in neuronal migration, construction of brain structure, and synapse formation and stability during the neurodevelopmental period. ${ }^{20}$ Schizophrenia is considered a result of abnormal neurodevelopment; abnormal expression of genes involved in neurodevelopment may contribute to its pathogenesis. ${ }^{21}$ Indeed, dysfunction of the

Table 1. Comparison of rs2073559, rs2229864, rs362691, and rs736707 genotypes between the schizophrenia and healthy control groups

\begin{tabular}{|c|c|c|c|c|c|c|c|c|c|c|c|c|c|}
\hline \multirow[t]{2}{*}{ Group } & \multirow[t]{2}{*}{ Sex } & \multicolumn{3}{|c|}{ rs2073559 } & \multicolumn{3}{|c|}{ rs2229864 } & \multicolumn{3}{|c|}{ rs362691 } & \multicolumn{3}{|c|}{ rs736707 } \\
\hline & & $\mathrm{CC}$ & TC & TT & $\mathbf{A A}$ & GA & GG & $\mathrm{CC}$ & CG & GG & $\mathbf{A A}$ & GA & GG \\
\hline Schizophrenia & Male $(n=92)$ & 32 & 51 & 9 & 5 & 28 & 59 & 0 & 21 & 71 & 27 & 49 & 16 \\
\hline Healthy control & Male $(\mathrm{n}=38)$ & 10 & 20 & 8 & 2 & 16 & 20 & 0 & 4 & 34 & 14 & 16 & 8 \\
\hline p Value & & \multicolumn{3}{|c|}{0.197} & \multicolumn{3}{|c|}{0.434} & \multicolumn{3}{|c|}{0.081} & \multicolumn{3}{|c|}{0.511} \\
\hline Schizophrenia & Female $(n=42)$ & 13 & 14 & 15 & 3 & 14 & 25 & 1 & 7 & 34 & 15 & 20 & 7 \\
\hline Healthy control & Female $(n=26)$ & 10 & 10 & 6 & 2 & 10 & 14 & 0 & 7 & 19 & 11 & 12 & 3 \\
\hline p Value & & \multicolumn{3}{|c|}{0.544} & \multicolumn{3}{|c|}{0.897} & \multicolumn{3}{|c|}{0.457} & \multicolumn{3}{|c|}{0.788} \\
\hline p Value & $\begin{array}{l}\text { Schizophrenia vs } \\
\text { healthy control }\end{array}$ & \multicolumn{3}{|c|}{0.798} & \multicolumn{3}{|c|}{0.415} & \multicolumn{3}{|c|}{0.642} & \multicolumn{3}{|c|}{0.524} \\
\hline
\end{tabular}

Table 2. Comparison of allele frequency of rs2073559, rs2229864, rs362691, and rs736707 between the schizophrenia and healthy control groups

\begin{tabular}{|c|c|c|c|c|c|c|c|c|c|}
\hline \multirow[t]{2}{*}{ Group } & \multirow[t]{2}{*}{ Sex } & \multicolumn{2}{|c|}{ rs2073559 } & \multicolumn{2}{|c|}{ rs2229864 } & \multicolumn{2}{|c|}{ rs362691 } & \multicolumn{2}{|c|}{ rs736707 } \\
\hline & & C & $\mathbf{T}$ & $\mathbf{A}$ & $\mathbf{G}$ & $\mathbf{C}$ & G & $\mathbf{A}$ & G \\
\hline Schizophrenia & Male $(\mathrm{n}=92)$ & 115 & 69 & 38 & 146 & 21 & 163 & 103 & 91 \\
\hline Healthy control & Male $(\mathrm{n}=38)$ & 40 & 36 & 20 & 56 & 4 & 72 & 44 & 32 \\
\hline \multicolumn{2}{|l|}{ p Value } & \multicolumn{2}{|c|}{0.091} & \multicolumn{2}{|c|}{0.201} & \multicolumn{2}{|c|}{0.093} & \multicolumn{2}{|c|}{0.283} \\
\hline Schizophrenia & Female $(\mathrm{n}=42)$ & 40 & 44 & 20 & 64 & 9 & 75 & 50 & 34 \\
\hline Healthy control & Female $(\mathrm{n}=26)$ & 30 & 22 & 14 & 38 & 7 & 45 & 34 & 18 \\
\hline p Value & & \multicolumn{2}{|c|}{0.167} & \multicolumn{2}{|c|}{0.416} & \multicolumn{2}{|c|}{0.411} & \multicolumn{2}{|c|}{0.309} \\
\hline p Value & $\begin{array}{l}\text { Schizophrenia vs } \\
\text { healthy control }\end{array}$ & \multicolumn{2}{|c|}{0.314} & \multicolumn{2}{|c|}{0.169} & \multicolumn{2}{|c|}{0.272} & \multicolumn{2}{|c|}{0.157} \\
\hline
\end{tabular}


Table 3. Haplotype association analysis

\begin{tabular}{|c|c|c|c|c|}
\hline \multirow[t]{2}{*}{ Haplotype } & \multicolumn{2}{|c|}{ No. (frequency) } & \multirow[t]{2}{*}{ p Value } & \multirow{2}{*}{$\begin{array}{c}\text { Odds ratio (95\% } \\
\text { confidence interval) }\end{array}$} \\
\hline & Schizophrenia & Healthy control & & \\
\hline \multicolumn{5}{|c|}{ rs2073559/rs2229864 } \\
\hline $\mathrm{CA}^{*}$ & $33(0.125)$ & $13(0.102)$ & 0.505 & $1.259(0.639-2.480)$ \\
\hline $\mathrm{CG}^{*}$ & $122(0.453)$ & $57(0.445)$ & 0.875 & $1.035(0.677-1.580)$ \\
\hline $\mathrm{TA}^{*}$ & $25(0.092)$ & $21(0.164)$ & 0.035 & $0.514(0.275-0.916)$ \\
\hline $\mathrm{TG}^{*}$ & $88(0.330)$ & $37(0.289)$ & 0.414 & $1.211(0.765-1.916)$ \\
\hline \multicolumn{5}{|c|}{ rs2073559/rs362691 } \\
\hline $\mathrm{CC}^{*}$ & $26(0.098)$ & $11(0.086)$ & 0.669 & $1.175(0.562-2.457)$ \\
\hline $\mathrm{CG}^{*}$ & $129(0.480)$ & $59(0.461)$ & 0.628 & $1.110(0.727-1.695)$ \\
\hline $\mathrm{TG}^{*}$ & $109(0.408)$ & $58(0.453)$ & 0.457 & $0.851(0.556-1.302)$ \\
\hline \multicolumn{5}{|c|}{ rs2073559/rs736707 } \\
\hline $\mathrm{CA}^{*}$ & $92(0.345)$ & $42(0.326)$ & 0.708 & $1.089(0.696-1.704)$ \\
\hline $\mathrm{CG}^{*}$ & $63(0.234)$ & $28(0.221)$ & 0.783 & $1.074(0.648-1.777)$ \\
\hline $\mathrm{TA}^{*}$ & $61(0.226)$ & $36(0.284)$ & 0.213 & $0.738(0.457-1.192)$ \\
\hline $\mathrm{TG}^{*}$ & $52(0.195)$ & $22(0.169)$ & 0.534 & $1.191(0.686-2.068)$ \\
\hline \multicolumn{5}{|c|}{ rs2229864/rs362691 } \\
\hline $\mathrm{AG}^{*}$ & $53(0.199)$ & $34(0.265)$ & 0.159 & $0.702(0.428-1.150)$ \\
\hline $\mathrm{GC}^{*}$ & $25(0.094)$ & $11(0.086)$ & 0.744 & $1.132(0.538-2.379)$ \\
\hline $\mathrm{GG}^{*}$ & $185(0.689)$ & $83(0.649)$ & 0.290 & $1.274(0.813-1.996)$ \\
\hline \multicolumn{5}{|c|}{ rs2229864/rs736707 } \\
\hline $\mathrm{AA}^{*}$ & $55(0.206)$ & $34(0.266)$ & 0.201 & $0.726(0.444-1.187)$ \\
\hline $\mathrm{GA}^{*}$ & $98(0.365)$ & $44(0.344)$ & 0.623 & $1.117(0.718-1.738)$ \\
\hline $\mathrm{GG}^{*}$ & $112(0.418)$ & $50(0.391)$ & 0.542 & $1.143(0.743-1.759)$ \\
\hline \multicolumn{5}{|c|}{ rs362691/rs736707 } \\
\hline $\mathrm{CA}^{*}$ & $17(0.063)$ & $8(0.059)$ & 0.880 & $1.071(0.441-2.598)$ \\
\hline $\mathrm{GA}^{*}$ & $136(0.508)$ & $70(0.551)$ & 0.429 & $0.843(0.553-1.287)$ \\
\hline $\mathrm{GG}^{*}$ & $102(0.380)$ & $47(0.364)$ & 0.753 & $1.073(0.693-1.660)$ \\
\hline \multicolumn{5}{|c|}{ rs2073559/rs2229864/rs362691 } \\
\hline $\mathrm{CAG}^{*}$ & $28(0.106)$ & $12(0.093)$ & 0.630 & $1.192(0.584-2.430)$ \\
\hline $\mathrm{CGC}^{*}$ & $21(0.080)$ & $10(0.086)$ & 0.914 & $0.959(0.448-2.052)$ \\
\hline $\mathrm{CGG}^{*}$ & $100(0.374)$ & $47(0.368)$ & 0.729 & $1.080(0.698-1.674)$ \\
\hline $\mathrm{TAG}^{*}$ & $25(0.092)$ & $21(0.164)$ & 0.028 & $0.503(0.271-0.934)$ \\
\hline TGG* & $85(0.317)$ & $36(0.281)$ & 0.351 & $1.247(0.784-1.985)$ \\
\hline \multicolumn{5}{|c|}{ rs2073559/rs2229864/rs736707 } \\
\hline $\mathrm{CAA}^{*}$ & $29(0.109)$ & $13(0.101)$ & 0.765 & $1.111(0.556-2.220)$ \\
\hline CGA* & $62(0.235)$ & $28(0.221)$ & 0.708 & $1.101(0.665-1.824)$ \\
\hline $\mathrm{CGG}^{*}$ & $60(0.223)$ & $29(0.226)$ & 0.998 & $1.000(0.604-1.657)$ \\
\hline TAA* & $25(0.092)$ & $21(0.165)$ & 0.054 & $0.547(0.295-1.016)$ \\
\hline TGA* & $35(0.130)$ & $16(0.123)$ & 0.826 & $1.074(0.568-2.030)$ \\
\hline $\mathrm{TGG}^{*}$ & $523(0.195)$ & $21(0.165)$ & 0.440 & $1.245(0.714-2.171)$ \\
\hline \multicolumn{5}{|c|}{ rs2073559/rs362691/rs736707 } \\
\hline $\mathrm{CCA}^{*}$ & $17(0.064)$ & $8(0.059)$ & 0.828 & $1.103(0.455-2.673)$ \\
\hline CGA* & $76(0.280)$ & $34(0.269)$ & 0.749 & $1.080(0.673-1.734)$ \\
\hline $\mathrm{CGG}^{*}$ & $53(0.199)$ & $25(0.192)$ & 0.822 & $1.063(0.624-1.810)$ \\
\hline TGA* & $60(0.227)$ & $36(0.282)$ & 0.263 & $0.761(0.471-1.229)$ \\
\hline TGG* & $49(0.182)$ & $22(0.171)$ & 0.758 & $1.091(0.626-1.901)$ \\
\hline \multicolumn{5}{|c|}{ rs2229864/rs362691/rs736707 } \\
\hline AGA* & $50(0.188)$ & $34(0.265)$ & 0.106 & $0.663(0.403-1.093)$ \\
\hline $\mathrm{GCA}^{*}$ & $12(0.045)$ & $7(0.051)$ & 0.837 & $0.903(0.341-2.393)$ \\
\hline $\mathrm{GCG}^{*}$ & $13(0.049)$ & $4(0.034)$ & 0.473 & $1.496(0.495-4.524)$ \\
\hline GGA* & $86(0.320)$ & $37(0.292)$ & 0.464 & $1.188(0.750-1.882)$ \\
\hline $\mathrm{GGG}^{*}$ & $99(0.370)$ & $46(0.357)$ & 0.648 & $1.108(0.714-1.720)$ \\
\hline \multicolumn{5}{|c|}{ rs2073559/rs2229864/rs362691/ } \\
\hline rs736707 & & & & \\
\hline CAGA* & $24(0.089)$ & $12(0.093)$ & 0.982 & $1.008(0.485-2.095)$ \\
\hline $\mathrm{CGCA}^{*}$ & $12(0.044)$ & $7(0.051)$ & 0.838 & $0.903(0.339-2.406)$ \\
\hline $\mathrm{CGCG}^{*}$ & $9(0.034)$ & $3(0.035)$ & 0.952 & $1.036(0.327-3.278)$ \\
\hline CGGA* & $50(0.191)$ & $22(0.173)$ & 0.537 & $1.189(0.685-2.064)$ \\
\hline $\mathrm{CGGG}^{*}$ & $50(0.190)$ & $25(0.196)$ & 0.951 & $1.017(0.596-1.734)$ \\
\hline TAGA* & $25(0.092)$ & $21(0.164)$ & 0.046 & $0.540(0.293-0.995)$ \\
\hline TGGA* & $34(0.130)$ & $15(0.120)$ & 0.661 & $1.154(0.607-2.194)$ \\
\hline TGGG* & $49(0.181)$ & $21(0.160)$ & 0.478 & $1.227(0.697-2.162)$ \\
\hline
\end{tabular}


Table 4. Associations between single-nucleotide polymorphism genotypes and quantitative traits in the Positive and Negative Syndrome Scale (PANSS) of the schizophrenia group

\begin{tabular}{|c|c|c|c|c|c|c|c|c|}
\hline \multirow{2}{*}{$\begin{array}{l}\text { PANSS } \\
\text { item }\end{array}$} & \multicolumn{2}{|c|}{ rs2073559 } & \multicolumn{2}{|c|}{ rs2229864 } & \multicolumn{2}{|c|}{ rs362691 } & \multicolumn{2}{|c|}{ rs736707 } \\
\hline & $\chi^{2}$ & p Value & $\chi^{2}$ & p Value & $\mathbf{Z}$ & p Value & $\chi^{2}$ & p Value \\
\hline $\mathrm{P} 1$ & 2.088 & 0.352 & 1.574 & 0.455 & 0.320 & 0.749 & 1.709 & 0.426 \\
\hline $\mathrm{P} 2$ & 0.290 & 0.865 & 0.569 & 0.752 & 1.205 & 0.228 & 1.483 & 0.476 \\
\hline $\mathrm{P} 3$ & 1.695 & 0.429 & 11.184 & 0.004 & 0.482 & 0.630 & 6.683 & 0.035 \\
\hline $\mathrm{P} 4$ & 2.171 & 0.338 & 0.085 & 0.958 & 0.650 & 0.516 & 1.395 & 0.498 \\
\hline P5 & 2.473 & 0.290 & 2.769 & 0.250 & 0.521 & 0.620 & 1.942 & 0.379 \\
\hline P6 & 0.546 & 0.761 & 2.177 & 0.337 & 0.669 & 0.503 & 4.588 & 0.101 \\
\hline $\mathrm{P} 7$ & 1.291 & 0.524 & 0.707 & 0.702 & 0.353 & 0.724 & 2.288 & 0.319 \\
\hline $\mathrm{N} 1$ & 1.652 & 0.438 & 1.192 & 0.551 & 1.548 & 0.122 & 0.005 & 0.997 \\
\hline $\mathrm{N} 2$ & 3.570 & 0.168 & 2.965 & 0.227 & 1.001 & 0.317 & 0.245 & 0.884 \\
\hline N3 & 3.016 & 0.221 & 0.217 & 0.897 & 0.585 & 0.558 & 0.838 & 0.658 \\
\hline N4 & 3.633 & 0.163 & 2.176 & 0.337 & 1.372 & 0.170 & 0.788 & 0.675 \\
\hline N5 & 1.455 & 0.483 & 0.830 & 0.660 & 1.478 & 0.139 & 1.083 & 0.582 \\
\hline N6 & 0.523 & 0.770 & 1.064 & 0.587 & 1.211 & 0.226 & 0.689 & 0.708 \\
\hline $\mathrm{N} 7$ & 1.298 & 0.522 & 2.404 & 0.301 & 0.643 & 0.521 & 0.091 & 0.955 \\
\hline G1 & 2.298 & 0.317 & 0.884 & 0.643 & 1.281 & 0.200 & 0.079 & 0.961 \\
\hline G2 & 1.232 & 0.540 & 1.371 & 0.504 & 1.134 & 0.257 & 0.008 & 0.996 \\
\hline G3 & 0.292 & 0.864 & 2.436 & 0.296 & 0.629 & 0.529 & 3.190 & 0.203 \\
\hline G4 & 0.067 & 0.967 & 1.010 & 0.604 & 1.127 & 0.260 & 2.139 & 0.343 \\
\hline G5 & 0.649 & 0.723 & 0.508 & 0.776 & 1.260 & 0.208 & 0.640 & 0.739 \\
\hline G6 & 0.453 & 0.797 & 0.008 & 0.996 & 0.480 & 0.631 & 0.650 & 0.723 \\
\hline G7 & 0.147 & 0.929 & 1.665 & 0.435 & 1.654 & 0.098 & 0.888 & 0.643 \\
\hline G8 & 3.818 & 0.148 & 2.277 & 0.320 & 0.297 & 0.767 & 0.087 & 0.957 \\
\hline G9 & 0.843 & 0.656 & 4.686 & 0.096 & 0.187 & 0.851 & 1.941 & 0.379 \\
\hline G10 & 1.942 & 0.379 & 1.219 & 0.544 & 2.162 & 0.041 & 0.182 & 0.913 \\
\hline G11 & 0.008 & 0.996 & 6.475 & 0.039 & 0.220 & 0.826 & 7.358 & 0.035 \\
\hline G12 & 4.292 & 0.117 & 1.869 & 0.393 & 1.474 & 0.140 & 0.133 & 0.936 \\
\hline G13 & 0.060 & 0.970 & 0.978 & 0.613 & 0.000 & 1.000 & 1.534 & 0.464 \\
\hline G14 & 0.213 & 0.899 & 0.751 & 0.687 & 0.609 & 0.542 & 0.487 & 0.784 \\
\hline G15 & 0.424 & 0.809 & 3.433 & 0.180 & 1.324 & 0.186 & 0.329 & 0.848 \\
\hline G16 & 0.552 & 0.759 & 3.228 & 0.199 & 0.410 & 0.682 & 0.562 & 0.755 \\
\hline
\end{tabular}

reelin signalling pathway has been found in people with schizophrenia. ${ }^{22}$ Our results provide further evidence for the hypothesis that deficits of neurodevelopment are the underlying mechanisms of schizophrenia.

According to our genetic risk analysis, rs2073559, rs2229864, rs362691, and rs736707, which are SNPs in RELN, showed no significant association with schizophrenia. Similarly, no significant association was found between the rs2073559, rs2229864, rs362691, and rs736707 in 165 autistic trios, 67 sporadically autistic children, and 283 healthy controls of Han Chinese ethnicity. ${ }^{23}$ In a study of RELN between patients with schizophrenia or bipolar disorder and controls, no association was found between rs2229864 and RELN gene expression, but allele disequilibrium expression of rs2229864 in patients with schizophrenia was observed, ${ }^{24}$ which is consistent with 
the findings of the present study. In a meta-analysis of $R E L N$ SNPs and putatively RELN-linked neuropsychiatric disorders (schizophrenia, autistic spectrum disorders, attention-deficit hyperactivity disorder, Alzheimer disease, and bipolar disorders), no association was found between the SNPs (rs2073559, rs2229864, rs362691) and the disorders. ${ }^{17}$ However, RELN rs736707 was significantly associated with schizophrenia in an Asian group. ${ }^{17}$ This is not consistent with our findings. The discrepancy may be related to sample size, which affects the estimation accuracy in populations with high genetic diversity.

The present study revealed strong linkage disequilibrium between rs2073559 and rs362691 and between rs2229864 and rs736707. Consistently, two studies also revealed strong linkage disequilibrium between rs736707 and rs2229864 in a Chinese population. ${ }^{23,25}$ Further studies are warranted to determine the functional interaction among rs736707 and other gene polymorphisms, such as rs2229864, in patients with schizophrenia.

Our findings suggest that the investigated haplotypes might be associated with schizophrenia, but the SNPs of RELN were not associated with the pathogenesis of schizophrenia after Bonferroni correction. Although RELN is $160 \mathrm{~kb}$ long and consists of 65 exons, only four SNPs that are evenly distributed across $R E L N$ were selected for the genetic polymorphism analysis. The small number of selected loci may limit the extent to which the results are indicative of the whole gene. Therefore, additional SNPs should be included for analysis to achieve more representative and reliable information about the association between RELN and schizophrenia. In addition, the sample size was small.

\section{Conclusion}

The SNPs rs2073559, rs2229864, rs362691, and rs736707 of RELN were not associated with the pathogenesis of schizophrenia. However, three of them (rs2229864, rs362691, and rs736707) might affect the clinical manifestations of schizophrenia. Considering the strong linkage disequilibrium (0.923) between rs2229864 and rs736707, we speculate that the haplotype composed of rs2229864-rs736707 is associated with hallucinatory behaviour and attention impairment in patients with schizophrenia.

\section{Contributors}

BGD and TYJ designed the study. JW, CYH, and JMY acquired the data. JJP and JZ analysed the data, drafted the manuscript, and critically revised the manuscript for important intellectual content. AB critically revised the manuscript. All authors had full access to the data, contributed to the study, approved the final version for publication, and take responsibility for its accuracy and integrity.

\section{Conflicts of Interest}

All authors have disclosed no conflicts of interest.

\section{Funding}

This study received funding from Social Welfare Science and Technology Research Project in Zhongshan (No.2019B1107, No.2018B1033) and Social Welfare Science and Technology Research Major Project in Zhongshan (No.2018B1006).

\section{Data Availability}

All data generated or analysed during the present study are available from the corresponding author on reasonable request.

\section{Ethics Approval}

The study was approved by the ethics committee of the Third People's Hospital of Zhongshan (reference: SSYLL20180301). The patients were treated in accordance with the tenets of the Declaration of Helsinki. The participants or their guardians provided written informed consent for all treatments and procedures and for publication.

\section{Acknowledgements}

We thank all participants for their support and participation and all staff for their contribution in clinical evaluation and blood sample collection.

\section{References}

1. Charlson FJ, Ferrari AJ, Santomauro DF, et al. Global epidemiology and burden of schizophrenia: findings from the Global Burden of Disease Study 2016. Schizophr Bull 2018;44:1195-203. Crossref

2. Huang Y, Wang Y, Wang H, et al. Prevalence of mental disorders in China: a cross-sectional epidemiological study. Lancet Psychiat 2019;6:211-24. Crossref

3. GBD 2015 Disease and Injury Incidence and Prevalence Collaborators. Global, regional, and national incidence, prevalence, and years lived with disability for 310 diseases and injuries, 1990-2015: a systematic analysis for the Global Burden of Disease Study 2015. Lancet 2016;388:1545-602. Crossref

4. Hailemichael Y, Hailemariam D, Tirfessa K, et al. Catastrophic outof-pocket payments for households of people with severe mental disorder: a comparative study in rural Ethiopia. Int J Mental Health Syst 2019;13:39. Crossref

5. Lichtenstein P, Yip BH, Björk C, et al. Common genetic determinants of schizophrenia and bipolar disorder in Swedish families: a populationbased study. Lancet 2009;373:234-9. Crossref

6. Hennah W, Thomson P, McQuillin A, et al. DISC1 association, heterogeneity and interplay in schizophrenia and bipolar disorder. Mol Psychiatry 2009;14:865-73. Crossref

7. Marshall CR, Howrigan DP, Merico D, et al. Contribution of copy number variants to schizophrenia from a genome-wide study of 41,321 subjects. Nat Genet 2017;49:27-35. Crossref

8. Walsh T, Mccletlan JM, Mccarthy SE, et al. Rare structural 
variants disrupt multiple genes in neurodevelopmental pathways in schizophrenia. Science 2008;320:539-43. Crossref

9. Need AC, Ge D, Weale ME, et al. A genome-wide investigation of SNPs and CNVs in schizophrenia. PLoS Genet 2009;5:e1000373. Crossref

10. Wedenoja J, Loukola A, Tuulio-Henriksson A, et al. Replication of linkage on chromosome 7q22 and association of the regional reelin gene with working memory in schizophrenia families. Mol Psychiatry 2008;13:673-84. Crossref

11. Shifman S, Johannesson M, Bronstein M, et al. Genome-wide association identifies a common variant in the reelin gene that increases the risk of schizophrenia only in women. PLoS Genet 2008;4:e28. Crossref

12. Zhou Z, Hu Z, Zhang L, et al. Identification of RELN variation p.Thr3192Ser in a Chinese family with schizophrenia. Sci Rep 2016;6:24327. Crossref

13. Li M, Luo XJ, Xiao X, et al. Analysis of common genetic variants identifies RELN as a risk gene for schizophrenia in Chinese population. World J Biol Psychiatry 2013;14: 91-9. Crossref

14. Kuang WJ, Sun RF, Zhu YS, Li SB. A new single-nucleotide mutation (rs362719) of the reelin (RELN) gene associated with schizophrenia in female Chinese Han. Genet Mol Res 2011;10:1650-8. Crossref

15. Folsom TD, Fatemi SH. The involvement of reelin in neurodevelopmental disorders. Neuropharmacology 2013;68:12235. Crossref

16. Ekelund J, Lichtermann D, Hovatta I, et al. Genome-wide scan for schizophrenia in the Finnish population: evidence for a locus on chromosome 7q22. Hum Mol Genet 2000;9:1049-57. Crossref

17. Chen N, Bao Y, Xue Y, et al. Meta-analyses of RELN variants in neuropsychiatric disorders. Behav Brain Res 2017;332:110-9. Crossref

18. Luo X, Chen S, Xue L, Chen JH, Shi YW, Zhao H. SNP Variation of RELN gene and schizophrenia in a Chinese population: a hospitalbased case-control study. Front Genet 2019;10:175. Crossref

19. Bai W, Fu Y, Yu X, et al. Association between RELN polymorphisms and schizophrenia in a Han population from Northeast China. Psychiatr Genet 2019;29:232-6. Crossref

20. Akbarian S, Bunney WE Jr, Potkin SG, et al. Altered distribution of nicotinamide-adenine dinucleotide phosphate-diaphorase cells in frontal lobe of schizophrenics implies disturbances of cortical development. Arch Gen Psychiatry 1993;50:169-77. Crossref

21. Hakak Y, Walker JR, Li C, et al. Genome-wide expression analysis reveals dysregulation of myelination-related genes in chronic schizophrenia. Proc Natl Acad Sci U S A 2001;98:4746-51. Crossref

22. Fatemi SH. Reelin glycoprotein in autism and schizophrenia. Int Rev Neurobiol 2005;71:179-87. Crossref

23. He Y, Xun G, Xia K, et al. No significant association between RELN polymorphism and autism in case-control and family-based association study in Chinese Han population. Psychiatry Res 2011;187:462-4. Crossref

24. Ovadia G, Shifman S. The genetic variation of RELN expression in schizophrenia and bipolar disorder. PLoS One 2011;6:e19955. Crossref

25. Li H, Li Y, Shao J, et al. The association analysis of RELN and GRM8 genes with autistic spectrum disorder in Chinese Han population. Am J Med Genet B Neuropsychiatr Genet 2008;147b:194-200. Crossref 